Int. J. Electrochem. Sci., 14 (2019) 7088 - 7103

\title{
A Sandwich-Structure Composite Membrane as Separator with High Wettability and Thermal Properties for Advanced Lithium-Ion Batteries
}

\author{
Yang $\mathrm{Li}^{1,2}$, Jiang $\mathrm{Cao}^{2,3, *}$, Qi $\mathrm{Liu}^{2}$, Aoxuan Wang ${ }^{1, *}$, Baohua $\mathrm{Li}^{2}$ \\ ${ }^{1}$ State Key Laboratory of Chemical Engineering, School of Chemical Engineering and Technology, \\ Tianjin University, Tianjin, 300350, China \\ ${ }^{2}$ Division of Energy and Environment, Engineering Laboratory for the Next Generation Power and \\ Energy Storage Batteries, Graduate School at Shenzhen, Tsinghua University, Shenzhen 518055, \\ China \\ ${ }^{3}$ R\&D Center, Shenzhen Senior Technology Material Co., LTD, Shenzhen 518106, China \\ *E-mail: aoxuanwang@tju.edu.cn, kos3123@163.com
}

doi: $10.20964 / 2019.08 .60$

Received: 2 April 2019 / Accepted: 31 May 2019 / Published: 30 June 2019

\begin{abstract}
Lithium-ion batteries with high energy density and environmental friendliness have attracted considerable interest for reseachers in recent years. However, the conventional polyolefin separators with poor electrolyte wettability and low thermal stability limited the further development of lithiumion batteries. Here we designed a porous poly(m-phenylene isophthalamide) (PMIA) layer, embedded with high-dispersed $\mathrm{TiO}_{2}-\mathrm{P}(\mathrm{MMA}-\mathrm{AA}-\mathrm{BA})$ (TP) nanohybrid, on both sides of a polyethylene (PE) membrane by a facile nonsolvent-induced phase inversion process to improve the electrolyte wettability and thermal stability of the PE separator. The TP nanoparticles can disperse uniformly in PMIA layer. Due to the inherent high-heat resistance and good affinity to the liquid electrolyte of PMIA and nanohybrid, this (TP-PMIA)-modified PE (TP-PMIA@PE) membrane exhibits high porosity, high ionic conductivity, improved thermal resistance and superior interfacial stability, which can endow the composite membrane-based $\mathrm{Li} / \mathrm{LiFePO}_{4}$ cell with good capacity retention and superior rate capability. The as-prepared composite membrane can serve as a promising separator for high-safety and highperformance lithium-ion batteries.
\end{abstract}

Keywords: Poly (m-phenylene isophthalamide); Nanohybrid ; Phase inversion process; Separator; Lithium-ion batteries 
(C) 2019 The Authors. Published by ESG (www.electrochemsci.org). This article is an open access article distributed under the terms and conditions of the Creative Commons Attribution license (http://creativecommons.org/licenses/by/4.0/). 